\title{
The Dynamic Rate-limiting Filtering Based on Preprocessing Sensor Data
}

\author{
Chao Tang, Zhou Wan \\ Faculty of Information Enginering and Automation, Kunming University of Science and Technology, Kunming, 650500,
} China

\begin{abstract}
Aiming at the noise inference when sensors testing data, this paper proposes a dynamic rate-limiting filtering algorithm to discover and eliminate the error. By the algorithm the author intends to preprocess the sensor output data to effectively eliminate the blunder error and ensure the data accurate and effective, thus improving the robustness of the overall system.
\end{abstract}

Keywords-noise interference; blunder error; the dynamic rate-limiting filtering.

\section{INTRODUCTION}

In industrial monitoring system, the need to use a large number of sensor, the sensor will be transmitted to the collected signals to the controller, the controller for signal processing and transmission to the actuator. In the process of system is the most important sensors, as sensors use after a long time, the external environment disturbance and other factors to cause a decline in sensor sensitivity and linearity, the collected data of the existence of inevitable blunder error, if you continue without dealing with the use of the collected with blunder error data, will make the system unstable, even may cause the system paralysis. Therefore, the sensor collected data preprocessing, eliminate noise, filtering the blunder error, improve system reliability and robustness, has very important practical significance.

The error in the measurement error of processing has done a lot of research, the literature [1] is used in arithmetic mean combined with recursive estimate method to deal with bizarre data processing, and the rule of Wright and literature [2] RON bass standards require data obey the normal distribution, and the practical application of the data is often difficult to obey statistical rule, which makes it impossible to effectively eliminate the obvious blunder error. Using histogram method in reference [3] to eliminate errors, the error data blunder error of this method in the multi-sensor data fusion processing has been widely used, it does not require data in accordance with the statistics law of a particular use effective interval to process the data, because this method can't reflect the characteristics of the data changes over time, lead to some outside the valid range of correct value be used as a singular data filtering.

Most of these blunder error processing method was a range of data, can't reflect the data time correlation, fully not found error point, there is a lot of disadvantages. Therefore, this article refer to limit method of digital filtering algorithm, and a timestamp, a dynamic speed limit filtering algorithm is proposed to handle blunder error, and eliminate the blunder error found in error has obtained the good effect.

\section{IMPLEMENTATION PROCESS OF THE ALGORITHM}

In dynamic speed limit filtering algorithm, if the input data values exceeded the given threshold, we think the value error error exists, if there is no more than the threshold, it need not to limit. Dynamic speed limit filtering algorithm is first measured values accord with certain probability distribution, calculated on the basis of this incredible distance measure, and combined with the timestamp of the acquisition data dynamically adjust speed limit threshold, such implementation is simple and applicable scope is wide, and can effectively overcome the accidental factors of pulse interference in order to compare to the same measurement parameters, the introduction of the concept of confidence distance measure, with probability distribution to describe the homogeneous sensor measurements, to compare between homogeneous sensors to get the data consistency, and possible error on the basis of test data.

As multi-sensor measures the same target parameter, Sensor No. $\mathrm{i}$ is set to $P_{i}(x)$, Sensor No. $\mathrm{j}$ is set to $P_{j}(x)$, and conditional probability functions are $P_{i j}=P_{i}\left(x_{j} \mid x_{i}\right)$ and $P_{j i}=P_{j}\left(x_{i} \mid x_{j}\right)$, then the same target parameter measured by these two multi-sensors is $d_{i j}$ :

$$
\begin{gathered}
d_{i j}=d_{j i}=\left|\int_{x_{i}}^{x_{j}} P_{i}\left(x \mid x_{i}\right) P_{i}\left(x_{i}\right) d x\right|+\left|\int_{x_{j}}^{x_{i}} P_{j}\left(x \mid x_{j}\right) P_{j}\left(x_{j}\right) d x\right| \\
\left|\int_{x_{i}}^{x_{j}} P_{i}\left(x \mid x_{i}\right) P_{i}\left(x_{i}\right) d x\right| \text { is the probability }
\end{gathered}
$$

distribution $P_{i}(x)$ in the area of between the two multisensor measurements $x_{i}$ and $x_{j}$, and $\left|\int_{x_{j}}^{x_{i}} P_{j}\left(x \mid x_{j}\right) P_{j}\left(x_{j}\right) d x\right| \quad$ is the probability distribution $P_{j}(x)$ in the contained area, even $0 \leq d_{i j} \leq 1$. Specially, if $d_{i j}=0, x_{i}$ is equal to $x_{j}$; while if $d_{i j}=1$, then $x_{i}$ and $x_{j}$ are quite different.

It is thus clear that the confidence interval measurement indicates probability distance, which reflects an extent of the two multi-sensors' measurement. The higher value $d_{i j}$ has, the more likely it is that the sensors in their 
measurements appear error; while the small value $d_{i j}$ indicates that the measurements of two sensors consistently reflects the true value of measured object.

The specific steps of the dynamic rate-limiting filtering algorithm are as follows:

Step1: According to the time order, measuring the samples for a while to get the sequence: $X_{1}, X_{2}, \cdots \cdots$, $X_{i}$;

Step2: Calculating the D-value between the two adjacent samples: $Y_{i} \quad, \quad Y_{1}=\left|X_{2}-X_{1}\right|$, $Y_{2}=\left|X_{3}-X_{2}\right|, \cdots \cdots, Y_{i}=\left|X_{i+1}-X_{i}\right|$;

Step3: Setting $\Delta$ the maximum deviation permissive between the two adjacent samples: $\Delta=k d_{i j} V_{a v g} T$. k is the filter factor of which the size is adjustable, depending on the processing precision of system; $V_{a v g}$ is the average rate of change of $X_{i}$; T is the sampling period.

Step4:If $Y_{i} \leq \Delta$, the error is allowable, and $X_{i}$ needs to be reserved; if $Y_{i}>\Delta$ which indicates the deviation between the two adjacent samples is too large, the timestamp $D_{T S_{i}}$ needs to be compared and the incremental amplitude threshold $\Delta^{\prime}$ needs to be recalculated: $\Delta^{\prime}=\Delta \frac{\left|D_{T S_{i+1}}-D_{T S_{i}}\right|}{T}$

If $Y_{i}>\Delta^{\prime}$, let the calculation of $Y_{i+1}$ continue; if $Y_{i+1} \leq \Delta$, let $X_{i}$ be the error point: $X_{i}=\frac{X_{i+1}+X_{i-1}}{2}$; if $Y_{i+1}>\Delta$ and $Y_{i+1}>\Delta^{\prime}, X_{i+1}$ will be judged to the error point: $X_{i+1}=\frac{X_{i+2}+X_{i}}{2}$; and if $Y_{i+1} \leq \Delta^{\prime}, X_{i}$ will the error point: $X_{i}=\frac{X_{i+1}+X_{i-1}}{2}$.

\section{PERFORMANCE ANALYSIS OF THE ALGORITHM}

Use the CYJ - 101 type of piezoresistive pressure sensor in continuous sampling data of a period of time as the experimental data, in order to better demonstrate the effectiveness of the algorithm, specially joined the impact error data in the data, select the appropriate filter coefficient $\mathrm{k}$, using Matlab programming algorithm model simulation, as shown in figure 1 of the filtering error error data before and after contrast figure.
From the comparison diagram below, there are impact errors of variable amplitude at the sampled time the 22nd second and the 299th second in the diagram with error data. Through the dynamic rate-limiting filtering algorithm, those errors have been identified correctly and filtered. There is still the mutation of data at the sampled time the 125th second. But the amplitude of the latter measurements is close to their adjacent ones'. By comparing their timestamps $D_{T S_{i}}$, the incremental amplitude threshold $\Delta^{\prime}$ needs to be recalculated and finally the data of this sampling point are judged not to be error information.
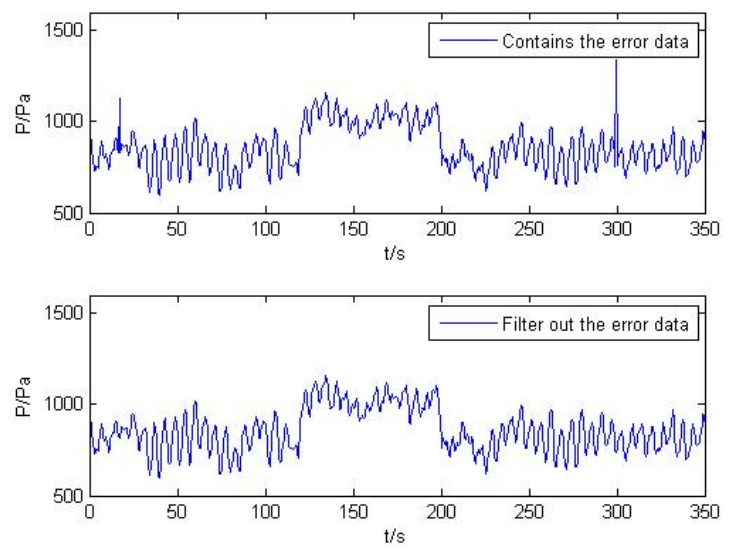

Figure 1. The comparison diagram before and after filtering error data

\section{IV.CONCLUSIONS}

Through the experiment test of pressure sensors' data, it can be seen that this algorithm combines the statistical rule with the existing time series, which can filter blunder error based on not only statistical rule, but also non-statistical rule. This algorithm can easily change the filtering accuracy only if it changes reference, thus having wider scope of application.

\section{REFERENCES}

[1] Zhang Chunjie,Si Xicai \& Xue Wei, Based on the arithmetic mean and recursive estimation of passive radar seeker data fusion processing. Applied Science and Technology,30 (3),pp.18-20 , 2003.

[2] Matviykiv Mykhaylo, Teslyuk Vasyl \& Matviykiv Taras. Prospects of using caibon film sensors for gas contamination monitoring in coal mines and drilling rigs. Modern Problems of Radio Engineering, Telecommunications and Computer Science-Proceedings of the 10th International Conference, pp .371,2010.

[3] Zhuo Jun, Histogram method in the application of error handling errors, Practical measurement technology,3(2),pp.33-35,2002.

[4] Liu Yelin \& Zhu Yanwei,The weighted data fusion algorithm and its application examples, Xian university of science and technology journals, 25 (2),pp.253-255,2005 